University of Nebraska - Lincoln

DigitalCommons@University of Nebraska - Lincoln

U.S. Air Force Research

U.S. Department of Defense

2006

\title{
Perchlorate Toxicity and Risk Assessment
}

David R. Mattie

Air Force Research Laboratoty

Joan Strawson

Toxicology Excellence for Risk Assessment

Jay Zhao

Toxicology Excellence for Risk Assessment

Follow this and additional works at: https://digitalcommons.unl.edu/usafresearch

Part of the Aerospace Engineering Commons

Mattie, David R.; Strawson, Joan; and Zhao, Jay, "Perchlorate Toxicity and Risk Assessment" (2006). U.S. Air Force Research. 7.

https://digitalcommons.unl.edu/usafresearch/7

This Article is brought to you for free and open access by the U.S. Department of Defense at DigitalCommons@University of Nebraska - Lincoln. It has been accepted for inclusion in U.S. Air Force Research by an authorized administrator of DigitalCommons@University of Nebraska - Lincoln. 


\title{
Perchlorate Toxicity and Risk Assessment
}

\author{
David R. Mattie, ${ }^{1}$ Joan Strawson, ${ }^{2}$ and Jay Zhao ${ }^{2}$ \\ ${ }^{1}$ Air Force Research Laboratory, WPAFB, OH 45433-5707 \\ ${ }^{2}$ Toxicology Excellence for Risk Assessment, Cincinnati, OH 45211
}

\section{PERCHLORATE OCCURRENCE AND EXPOSURE}

\section{Sources of Perchlorate}

Ammonium perchlorate is the oxidizer ingredient in solid propellant mixtures for rockets, missiles and munitions such as Titan, Minuteman, Peacekeeper, Hawk, Polaris and the Space Shuttle. Perchlorate salts may also be used in medicine, matches, munitions and pyrotechnics (illuminating and signaling flares, colored and white smoke generators, tracers, incendiary delays, fuses, photo-flash compounds and fireworks). Perchlorate is also found in lubricating oils, finished leather, fabric fixer, dyes, electroplating, aluminum refining, manufacture of rubber, paint and enamel production, as an additive in cattle feed, in magnesium batteries and as a component of automobile air bag inflators. ${ }^{1}$

\section{Exposure Routes/Pathways}

Perchlorate salts are highly water-soluble and fully ionize in water. ${ }^{2}$ The resulting perchlorate ion is identical whether it is from the ammonium salt or another salt such as potassium and its toxicity is due to the perchlorate ion $\left(\mathrm{ClO}_{4}{ }^{-}\right)$. Chemicals may enter the human body in several ways, known as routes of exposure or pathways. Routes of exposure can include ingestion, dermal (skin) absorption and inhalation. The primary route of exposure for perchlorate is through ingestion of water from contaminated drinking water supplies, although ingestion of contaminated food and milk are other potential sources of exposure.

Absorption through skin and inhalation of perchlorate are minor exposure pathways. Compounds most readily absorbed through the skin are primarily organic chemicals. Because perchlorate is an inorganic compound and 
completely ionized in water, the potential for dermal absorption of perchlorate through intact skin while bathing and washing is unlikely.

Occupational exposure of workers to perchlorate is primarily through inhalation of ammonium perchlorate dust during the commercial production or use of perchlorate salts. Occupational exposure in ammonium perchlorate production facilities was shown to be higher than potential exposures from drinking water or food sources. ${ }^{3,4}$ Exposure by inhalation results in absorption into the body through the mucous membranes in the respiratory and gastrointestinal tracts. Some direct ingestion through the oral route is possible, as is dermal contact, but both of these pathways are minor in occupational settings.

\section{PERCHLORATE HEALTH EFFECTS}

\section{Mechanism of Toxicity}

Thyroid hormones are essential to the regulation of oxygen consumption and metabolism throughout the body. Thyroid iodine metabolism and the levels of thyroid hormone in serum and tissues are regulated by a number of fairly well understood homeostatic mechanisms. ${ }^{5}$ Thyrotropin (TSH), a hormone synthesized and secreted by the anterior pituitary gland, is the primary regulator of thyroidal iodide uptake and other aspects of thyroid function. ${ }^{6}$ The secretion of TSH is regulated by a negative feed-back pathway through serum level of $T_{3}$ and $T_{4}$. Formation of thyroid hormones is depressed if sufficient inhibition of iodide uptake occurs. As a result, increased TSH secretion from the anterior pituitary gland will stimulate the thyroid to produce more $T_{3}$ and $T_{4}$. Inhibition of iodine uptake is the basis for the current and former pharmacological uses of perchlorate.

Perchlorate, like many chemicals and drugs, disrupts one or more steps in the synthesis and secretion of thyroid hormones, resulting in subnormal levels of $\mathrm{T}_{4}$ and $\mathrm{T}_{3}$ and an associated compensatory increase in secretion of TSH. ${ }^{7}$ The perchlorate ion, because of its similarity to iodide in ionic size and charge, competes with iodide for uptake into the thyroid gland by the sodium-iodide symporter, a transport mechanism in the membranes of thyroid cells. At high doses, this competitive inhibition results in reduced production of the thyroid hormones [triiodothyronine $\left(\mathrm{T}_{3}\right)$ and thyroxin $\left(\mathrm{T}_{4}\right)$ ] and a consequent increase in thyroid stimulating hormone (TSH) via a negative feedback loop involving the thyroid, pituitary and hypothalamus. ${ }^{8}$ Subsequent events include decreases in serum $T_{4}$ (and $T_{3}$ ), leading to the potential for altered neurodevelopment if observed in either mothers or fetuses/neonates, and increases in serum TSH, leading to the potential for thyroid hyperplasia and 
tumors. The repeated observation of thyroid effects such as alterations of hormones, increased thyroid weight, and alterations of thyroid histopathology (including tumors) from a large number of rat studies on perchlorate provide supporting evidence for the proposed mode-of-action, and confirms that the perturbation of thyroid hormone economy as the primary biological effect of perchlorate.

\section{Toxicokinetics}

Studies of absorption, distribution, metabolism and elimination (ADME) to measure perchlorate kinetics revealed that there was no metabolism of perchlorate in either adult rats or humans. Perchlorate is rapidly excreted, with urinary half-lives on the order of 4 hours in the rat and 6 hours in humans. ${ }^{9,10}$ Kinetics studies were also conducted for fetal and lactational time points in rats in parallel with the "Effects" Study. Kinetic studies were designed to aid quantitative interspecies extrapolation and form the basis for physiologically based pharmacokinetic (PBPK) models for adult rats and humans, as well as pregnant and lactating rats. ${ }^{11,12,13,14}$ The kinetic studies were also used to evaluate the mechanism of iodide inhibition by perchlorate and the subsequent hormone response. By determining the relative sensitivity of rat versus human, the results helped aid interspecies extrapolation. The results of PBPK model simulations confirmed that the fetus is the most sensitive developmental time point based on extent of iodide inhibition. ${ }^{13}$

\section{Animal Toxicity Studies}

In order to determine the health effects of perchlorate in humans, a fairly extensive database of animal toxicity studies was developed in keeping with risk assessment guidelines and the mechanism of action of perchlorate. Several studies of perchlorate in rodents have been conducted in which hormone measurements and thyroid histopathology have been evaluated. Data are available in male and female rats following 14 and 90 days of exposure, ${ }^{15,16}$ female mice following 90 days of exposure ${ }^{17,18}$ rat dams on gestation day 20 , post natal day 5 , postnatal day $10,9,19,20$ and male and female pups on post natal days 5,10 , and 22 . $^{9,19,20}$

A 90-day subchronic bioassay determined that the thyroid was the only target organ in male and female rats exposed to perchlorate in drinking water $(0$, $0.01,0.05,0.2,1.0$, and $10 \mathrm{mg} / \mathrm{kg}$-day) for 90 days. The no observable adverse effect level (NOAEL) based on thyroid changes was $1 \mathrm{mg} / \mathrm{kg}$-day but hormone changes, decreased $\mathrm{T}_{4}$ and increased TSH, were still seen at the lowest doses. ${ }^{16}$ 
Genotoxicity assays showed that perchlorate has no toxic effects on genes or chromosomes in cells. ${ }^{21}$ Perchlorate is not a teratogen as no birth defects were found at doses as high as $100 \mathrm{mg} / \mathrm{kg}$-day in the rabbit ${ }^{22}$ or as high as 30 $\mathrm{mg} / \mathrm{kg}$-day in the rat. ${ }^{23}$ Immunotoxicity studies were motivated by case reports of aplastic anemia and leukopenia in humans when perchlorate was used in high doses as an anti-thyroid drug. Studies using female mice did not demonstrate any adverse effects to the immune system. Evaluation of thyroid responses identified no alterations in $T_{3}$ and $T S H$, while $T_{4}$ was decreased after exposure to $1.0,3.0$ or $30 \mathrm{mg} / \mathrm{kg}$-day. Thyroid changes detected histologically were not seen in all animals until the $30.0 \mathrm{mg} / \mathrm{kg}$-day dose. ${ }^{17}$

Developmental neurotoxicity studies exposed pregnant rats to perchlorate in drinking water $(0,0.1,1.0,3.0$ and $10 \mathrm{mg} / \mathrm{kg}$-day) during pregnancy through day 10 of lactation. ${ }^{24}$ No pup behavioral effects were seen except marginal motor activity results at one time point. An additional motor activity study with the same doses found no statistically significant effects in motor activity. ${ }^{25,26}$ However, a Bayesian statistical analysis conducted using data from the two different motor activity studies combined resulted in a NOAEL of $1.0 \mathrm{mg} / \mathrm{kg}$-day. Hormone changes, decreased $\mathrm{T}_{4}$ and increased $\mathrm{TSH}$, were again seen at lower doses. Brain histology and morphometry changes seen in this developmental study were not dose dependent and resulted in larger measured values. Iodine deficiency should produce decreases in brain structure size. ${ }^{24}$ Another study was planned as a result of the brain data.

The additional major study performed was referred to as the "Effects" Study. The objectives were to refine understanding of the effects of perchlorate in the thyroid gland and to evaluate brain development at critical time points. ${ }^{19,20}$ The brain analysis attempted to provide better brain morphometry data than the developmental study described above. The study was designed to provide critical information for the perchlorate risk assessment, so data were collected at one fetal and three postnatal (during lactation) time points. The fetal time point also provided rat teratogenic results to compare with the rabbit teratogenic study. ${ }^{22,23}$ Hormone changes, decreased $\mathrm{T}_{4}$ and increased TSH, were again seen at lower doses. However, the brain morphometry was equivocal because the changes seen were once again not dose dependent or resulted in larger measured values. Subsequent evaluation of the methodology used in this and the previous developmental neurotoxicity raised questions about preparation of the brain tissue for microscopic analysis, including orientation of the slices through the brain. The interpretation of the brain morphometry data has been debated as a result. 
A two-generation reproductive toxicity study was used to evaluate fertility in adult rats and viability/toxicity in their offspring. ${ }^{27}$ Reproductive parameters were tested over two generations of drinking water exposure to perchlorate. The NOAEL for reproduction effects is greater than the highest dose tested, $30 \mathrm{mg} / \mathrm{kg}$-day. Thyroid histology changes were seen starting at $3 \mathrm{mg} / \mathrm{kg}$-day. There were three rare benign thyroid tumors identified by a Pathology Working Group in two first generation (F1) pups at the $30 \mathrm{mg} / \mathrm{kg}$-day dose. However, the occurrence of this tumor was not statistically significant as it was also found in control animals.

\section{Perchlorate Use as a Drug in Humans}

Potassium or sodium perchlorate was used clinically to treat Graves' disease (hyperthyroidism, thyrotoxicosis) in the 1950s and 1960s. Anti-thyroid drugs act by either blocking iodide uptake into the thyroid or by blocking thyroid hormone $\left(\mathrm{T}_{3}\right.$ and $\left.\mathrm{T}_{4}\right)$ synthesis in the thyroid. The recommended dose was 800 to $1000 \mathrm{mg} /$ day, although doses varied from 200 to 2000 $\mathrm{mg} /$ day. Perchlorate was given as either a single large dose or as a daily dose for weeks, months or even years in some cases. The use of perchlorate as a drug became limited in the mid-1960s due to reports of agranulocytosis and fatal aplastic anemia. ${ }^{28}$

More recently, amiodarone has been used to treat patients with ischemic heart disease or with ventricular tachycardia; this drug has a potential side effect of hyperthyroidism. A dose of $1000 \mathrm{mg}$ /day potassium perchlorate has been used to treat amiodarone-induced hyperthyroidism. Potassium perchlorate may also be used if a patient is sensitive to standard antithyroid drugs. The perchlorate discharge test was used clinically to diagnose thyroid function. In this test, a single large dose of perchlorate caused iodide discharge from the thyroid if there was a problem with the organification process, which is the incorporation of iodide into thyroid hormones. ${ }^{28}$

\section{Human Health Effects}

Epidemiological studies have examined the associations of thyroid effects and environmental exposure to perchlorate in public drinking water at levels ranging from 4 to $120 \mathrm{ppb}(4$ to $120 \mu \mathrm{g} / \mathrm{L}$ ). A number of the studies have small sample sizes and cannot detect differences in frequency of outcomes between exposure groups. Adjustments for potentially confounding factors were limited. Nearly all the studies were ecologic (epidemiological studies without individual exposure characterization), including those in newborns and children, the groups potentially most vulnerable to the effects of perchlorate exposure. Ecologic studies can provide supporting evidence of a possible association but cannot provide definitive evidence regarding cause. 
Perchlorate exposure of individual subjects is difficult to measure and was not assessed directly in any of the published studies conducted outside the occupational setting.

Perchlorate was found in Las Vegas and the surrounding county at levels averaging $14 \mathrm{ppb}(\mu \mathrm{g} / \mathrm{L})$. Congenital hypothyroidism data from the 1996 and 1997 neonatal screening program were examined; no increase in congenital hypothyroidism was observed. ${ }^{29}$ From April 1998 to June 1999, the monthly mean $\mathrm{T}_{4}$ levels of neonates from Las Vegas (an area with perchloratecontaminated drinking water at 9 to $15 \mathrm{ppb}(\mu \mathrm{g} / \mathrm{L})$ for eight months and nondetectable $(<4 \mathrm{ppb})$ contamination for 7 months) were compared with those of neonates from Reno (an area with no detectable perchlorate in its drinking water). There were no differences in neonatal $\mathrm{T}_{4}$ levels between the cities. ${ }^{30}$ An analysis of newborn TSH levels from Las Vegas and Reno was conducted for babies born December 1998 through October 1999 at normal birth weight and having their blood sampled within the first month of life. The mean blood TSH levels were not different between the cities. ${ }^{31}$ Another Las Vegas vs. Reno comparison examined thyroid disease prevalence rates among Medicaid-eligible residents. Thyroid disease included simple goiter, nodular goiter, thyrotoxicosis, congenital hypothyroidism, acquired hypothyroidism, thyroid cancer or other thyroid diseases. Again there were no differences between the cities. ${ }^{32}$ One study examined a possible relation between perchlorate exposure and adverse neurodevelopmental outcomes in children and did not see any association between perchlorate and attention deficit hyperactivity disorder (ADHD) or autism. ${ }^{33}$

Data on thyroid hormone and TSH production in newborns and congenital hypothyroidism were also examined by Brechner et al. ${ }^{34}$ Schwartz,${ }^{35}$ Kelsh et al. ${ }^{36}$ Lamm $^{37}$ and Buffler et al. ${ }^{38}$ Although hormone changes were reported in areas of California and Arizona, the prevalence of congenital hypothyroidism was not associated with perchlorate exposure. Morgan and Cassady $^{39}$ addressed the incidence of cancer after dual exposure to perchlorate and trichloroethylene. Incidence of cancers was not observed more often than expected in children.

A number of studies collected clinical data from adult human volunteers in a controlled setting after exposure to known amounts of perchlorate in drinking water ranging from 0.007 to $12 \mathrm{mg} / \mathrm{kg}$ per day. These clinical studies measured inhibition of iodide uptake into the thyroid glands as well as TSH and thyroid hormones. Serum TSH levels did not increase and thyroid hormones did not decrease in any group. Brabant et al. ${ }^{40}$ conducted a 4 week study with subjects given $12 \mathrm{mg} / \mathrm{kg}$ per day. Thyroid iodide and TSH decreased and thyroid hormones did not change. Although only preliminary data are available, a study in which volunteers were given 0.007 and 0.04 
$\mathrm{mg} / \mathrm{kg}$-day perchlorate for 6 months resulted in no effects on thyroid function. ${ }^{41}$

Two 14-day studies were conducted in which $10 \mathrm{mg}$ /day was provided in water to 10 male subjects and $3 \mathrm{mg} /$ day was provided in drinking water to 8 male subjects. ${ }^{42,43}$ In both studies, each subject served as their own control by having measurements taken before and after perchlorate consumption. Iodide ${ }^{123}$ was measured in the thyroid to obtain inhibition data, and iodide and perchlorate were determined in blood and urine. Perchlorate, at both 3 and $10 \mathrm{mg} /$ day, caused inhibition of iodide uptake into the thyroid $(38 \%$ and $10 \%$, respectively). There were no changes seen in TSH or thyroid hormone levels in the blood. The extrapolated no observable effect level (NOEL) for iodide inhibition was $2 \mathrm{mg} /$ day based on these two exposures.

Another 14-day study employed 10 subjects ( 5 male $/ 5$ female) for each dose $\left(0.5,0.1,0.02\right.$ and $0.007 \mathrm{mg} / \mathrm{kg}$-day) who also served as their own control. ${ }^{10}$ The parameters measured were iodide ${ }^{123}$ uptake in the thyroid for inhibition data and iodide and perchlorate in blood and urine for kinetic data. There were no changes seen in TSH or thyroid hormone levels in the blood. The NOEL for iodide inhibition, measured as decreased uptake of iodide ${ }^{123}$ in the thyroid, was $0.007 \mathrm{mg} / \mathrm{kg}$-day. Data from these studies were used to develop the human PBPK model for perchlorate.

Employees were examined at an ammonium perchlorate production facility in Nevada and their findings compared to those of a large unexposed control population from the same industrial complex. ${ }^{3}$ The average working-lifetime cumulative dose in the higher exposure group was estimated to be $38 \mathrm{mg} / \mathrm{kg}$. Based on both cumulative and single-shift perchlorate exposures, there were no adverse effects on thyroid, kidney, liver or bone marrow function. A cross-sectional health study of two similar worker populations, a group of ammonium perchlorate workers divided into three exposure groups and a comparison group of other workers from the same industrial complex, was conducted at a perchlorate manufacturing plant in Utah. ${ }^{44}$ More than $40 \%$ of the exposed employees had been working with perchlorate for more than five years. There were no effects on blood and clinical chemical parameters at any level of exposure up to $34 \mathrm{mg}$ per day.

A second occupational study of perchlorate exposure was conducted at the same site in Utah. Serum perchlorate levels averaged $838 \mathrm{ppb}(\mu \mathrm{g} / \mathrm{L})$ during exposure with a calculated daily dose of $0.167 \mathrm{mg} / \mathrm{kg}$-day perchlorate. Although iodide inhibition averaged $38 \%$, TSH did not change and thyroid hormones actually increased slightly but significantly. ${ }^{4}$ 
School-age children were examined in three cities in northern Chile where levels of naturally occurring perchlorate in the water supply were undetectable, 5-7 and 100-120 ppb (ug/L). In the school children (mean age 7.3 years), 127 of whom had lifelong residence in their respective cities, mean $\mathrm{TSH}, \mathrm{T}_{4}$, and $\mathrm{T}_{3}$, were similar among the three cities. Incidence of goiter in the lifelong residents was similar in all three cities; although the residents in Taltal self-reported a higher incidence of family history of thyroid disease. No changes were found in congenital hypothyroidism, and clinical differences between children from the three different cities. This was the only ecologic study with measures of perchlorate made directly from drinking-water samples taken from homes in the area where subjects lived. ${ }^{45}$ Free $T_{4}$ was significantly increased in children living in Taltal and Chanaral, compared with Antofagasta (control city), a change in the opposite direction than expected. A variable introduction of iodized salt started in 1982 and may have affected these observations. Crump et al. ${ }^{45}$ also studied newborns screened for hypothyroidism by a heel-stick blood sample between February 1996 and January 1999 in the same three Chilean cities. TSH levels were significantly lower in Taltal than in the other two cities, a trend opposite to that hypothesized. The authors concluded that the differences did not appear clinically significant.

A second epidemiological study was conducted in the same Chilean cities. Water was analyzed from the tap in the home of every subject. Perchlorate, at daily levels as high as $114 \mathrm{ppb}(\mu \mathrm{g} / \mathrm{L})$ throughout pregnancy, did not affect maternal thyroid status early in gestation, alter fetal thyroid status at birth, or reduce breast milk iodine concentrations. ${ }^{46}$

\section{Summary of Toxicity}

The competitive inhibition of iodide uptake is the only direct perchlorate effect on the thyroid, leading to a reversible chemical-induced iodine deficiency. Alteration of hormones $\left(\mathrm{T}_{4}, \mathrm{~T}_{3}, \mathrm{TSH}\right)$ would be the first observed biological effect of perchlorate exposure. Following a prolonged increase in $\mathrm{TSH}$, thyroid hyperplasia progressing to thyroid tumors would be expected to occur in rodents. However, the relevance of these tumors to humans has been questioned, since this progression has not been observed in humans. ${ }^{47}$ In contrast, human data show that decreased $\mathrm{T}_{4}$ levels, both in pregnant women and in neonates, can lead to neuro-developmental deficit; although this has not been confirmed in animals following perchlorate exposure. Therefore, of the two pathways to altered structure and function proposed by a mode-of-action analysis for perchlorate, decreased $T_{4}$ leading to potential neurodevelopmental effects is more relevant to an assessment of human health. 
This conclusion is also supported by National Research Council. ${ }^{48}$ The NRC, on behalf of the DoD, EPA, DOE and NASA, formed a committee in 2003 to conduct an assessment of key scientific issues associated with the health effects of perchlorate. This committee in its report ${ }^{48}$ judged that the development of thyroid tumors as an ultimate result of perchlorate exposure is an unlikely outcome in human based on two considerations: (1) rats are sensitive to the development of thyroid tumors because their thyroid function is easily disrupted; (2) humans are much less susceptible than rats to disruption of thyroid function and therefore are not likely to develop thyroid tumors as a result of perchlorate exposure. Therefore, NRC committee concluded that the most reasonable pathway of events after changes in thyroid hormone and TSH secretion would be thyroid hypertrophy or hyperplasia, possibly leading to hypothyroidism. ${ }^{48}$ The hypothyroidism would lead to two potential outcomes (as summarized in Figure 1): (1) metabolic sequelae, such as decreased metabolic rate and slowing of the function of many organ systems, occurring at any age, and (2) abnormal growth and development in fetuses and children.

Following oral exposure in drinking water, serum perchlorate levels increase and provide a measure of the perchlorate internal dose. The mechanism of action also identifies that perchlorate has a threshold for effects and that the degree of effects are dependent on the dosage. Severe or sustained iodine deficiency can lead to hypothyroidism. However, a temporary disruption of iodine leads to temporary effects that are reversed with the proper levels of iodine ingestion in the diet. In rodents, sustained exposure to a chemical that causes hypothyroidism can lead to thyroid tumors, a mechanism that has not been demonstrated in humans. There remains disagreement on what is the first adverse or critical effect after perchlorate exposure.

\section{PERCHLORATE RISK ASSESSMENT}

\section{Identification of critical effect}

One risk assessment goal is to determine what exposure might be considered "safe." "Safe" or subthreshold doses are defined by a number of health agencies worldwide. Although many of the underlying assumptions, judgments of critical effect, and choices of uncertainty factors are similar among health agencies in estimating these subthreshold doses, this section will follow U.S. EPA's Reference Dose (RfD) methods. ${ }^{49,50,51}$

The first step in defining an RfD is to identify the critical effect(s). U.S. EPA ${ }^{52}$ and Haber et al. ${ }^{53}$ define critical effect(s) as the first adverse 


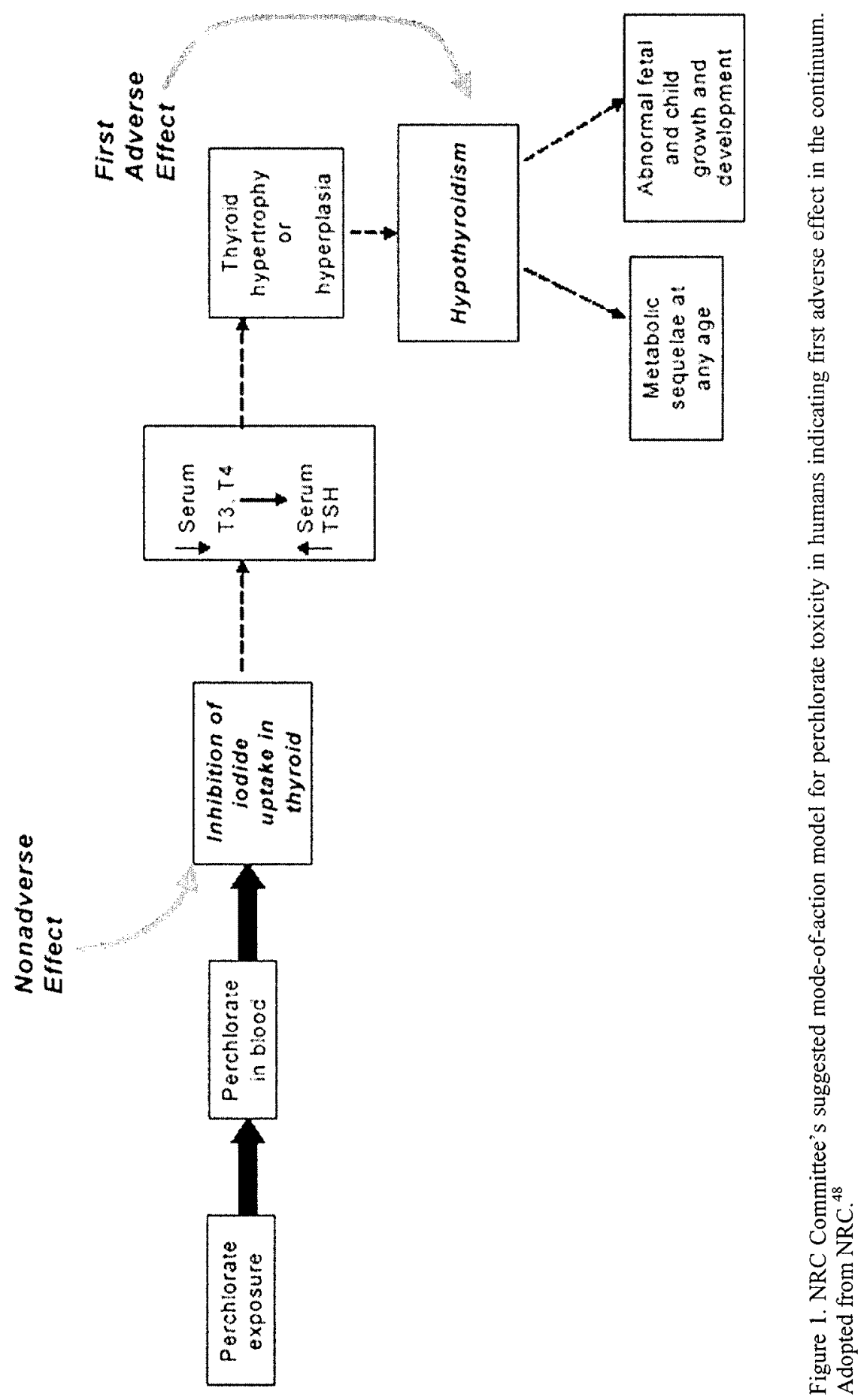


effect(s), or its known precursor, that occurs as dose rate or exposure level increases. In the determination of critical effect, it is crucial that distinctions be drawn between adverse effects and adaptive effects. An adaptive effect enhances an organism's performance as a whole and/or its ability to withstand a challenge; an adverse effect is a biochemical change, functional impairment, or pathological lesion that impairs performance and reduces the ability of an organism to respond to additional challenge. ${ }^{49,52}$ Available animal studies as described above clearly suggest that the thyroid is the primary target organ for perchlorate. Thus, distinguishing adaptive from adverse effects in the thyroid and determining the most appropriate adverse effect on which to base an RfD was the first step in the perchlorate risk assessment.

Inhibition of iodine uptake in thyroid, the key event in the ultimate disruption of thyroid function, can be considered as a marker of the biologically effective dose for perchlorate. However, inhibition of iodine uptake, itself, cannot be considered an adverse effect because in humans we do not yet know what levels of iodine uptake inhibition would decrease $T_{4}$ levels. Alteration of hormone levels, including decrease of serum $T_{4}$ and $T_{3}$ with a corresponding increase of TSH, is considered to be the early biological effect of exposure to perchlorate. The human body has a large reserve capacity of circulating thyroid hormone; serum levels of $T_{4}$ and $T_{3}$ are highly variable. No clear-cut information is available on how much decrement of circulating serum $\mathrm{T}_{4}$ can be tolerated without resulting in permanent alteration of thyroid function. However, subclinical hypothyroidism is generally considered to be present when circulating TSH levels are elevated by 2 -fold, with, or without decreased levels of $\mathrm{T}_{4}{ }^{54}$

Based on a mode-of-action analysis, it is clear that altered hormone levels are an early biological effect of perchlorate exposure. If allowed to persist, increased TSH levels, at least in rodents, will eventually lead to thyroid hyperplasia and possible thyroid tumors. Even if this pathway is not relevant to humans, persistent decreases in $T_{4}$ levels increase the potential for neurodevelopmental deficits in children. In this case, decreased $\mathrm{T}_{4}$ can be considered to be a precursor to an adverse effect, rather than an adverse effect in itself, however, because changes in $T_{4}$ are routinely compensated by normal, and well understood, homeostatic processes. Therefore, decreases in serum $\mathrm{T}_{4}$ in the pregnant population should be considered to be the critical effect most relevant to human health. The NRC committee ${ }^{48}$ also reached the same conclusion and considered that the first adverse effect in the model-ofaction model is hypothyroidism. 


\section{Choice of appropriate species and study}

US EPA's policy when developing RfDs in many of its program, regional offices, and Office of Research and Development (ORD) has been to use human data first and foremost in the determination of critical effect and choice of uncertainty factors. The preference for use of human data is found in many EPA publications, risk positions, risk methods documents, and practice. $^{49,51}$ In general, using human data as the basis for developing an RfD will reduce the uncertainty inherent in extrapolating from rat data.

The available data on the effects of perchlorate in experimental animals consistently points to thyroid disturbance as the sentinel effect. This disturbance may lead to subsequent thyroid and neurological damage. This information in experimental animals is consistent with the available, but more limited, human data. However, these data also demonstrate that rats may respond to perchlorate exposures in a very different manner than humans. ${ }^{55}$ The reason that such comparisons are not definitive is that the human data do not include information on pregnant individuals.

Although the rat data set includes the sensitive subgroup (the pregnant animal and its fetus), whereas the human studies only include measurements of TSH and $\mathrm{T}_{4}$ in adults, infants and children (and not pregnant individuals), rats are known to be more sensitive than humans to thyroid hormone replacement therapy, needing 10 times more $T_{4}$ than humans to achieve a euthyroid condition. ${ }^{56}$ Because the overall uncertainty in determining an RfD is greater from the rat data, when compared with the human data, the human data are the appropriate choice for determining an RfD. The $\mathrm{NRC}^{48}$ also concluded that the human data provided a more reliable point of departure for the risk assessment than the animal data.

Of the available human studies, one clinical study ${ }^{10}$ and one epidemiology study ${ }^{45}$ were considered to yield sufficient information to determine an RfD. Greer study was a well-conducted study underwent a rigorous quality assurance audit and conforms to the "Common Rule" the Federal Agency Guidelines on the ethical conduct of human studies. ${ }^{57}$ This study observed no statistically significant effects in serum $\mathrm{T}_{4}, \mathrm{~T}_{3}$, or TSH even at the highest dose tested, and defines a NOAEL of $0.5 \mathrm{mg} / \mathrm{kg}$-day for the healthy adult human population for short-term exposure. The Crump et al. ${ }^{45}$ study found no evidence that perchlorate in drinking water at concentrations as high as $120 \mathrm{ug} / \mathrm{L}$ suppressed thyroid function in newborns or school-aged children. Crump et $\mathrm{al}^{45}$ also studied newborns screened for hypothyroidism by a heelstick blood sample between February 1996 and January 1999 in the same three Chilean cities. TSH levels were significantly lower in Taltal (high exposure city) than in the other two cities, a trend opposite to that 
hypothesized. The authors concluded that the differences did not appear clinically significant.

One issue to address in the use of Crump et $a l .^{45}$ study as a basis of an RfD is the apparent iodine excess when compared with other populations, such as the U.S. A 1 to 2.5-fold excess in urinary iodine seen in the Chilean school children may serve to protect this population from perchlorate exposure. However, a second study with pregnant women in the same cities of Chile with lower iodine supplementation had the same results as the Crump et $a l^{45}$ study, ${ }^{46}$

\section{Point-of-departure analysis}

Following accepted risk assessment approaches, a point-of-departure analysis establishes the threshold dose that serves as the starting point for developing the RfD. Traditionally, the point of departure for an RfD has been the No Observed Adverse Effect Level (NOAEL), which is the highest experimental dose that is without adverse effect. More recently, risk assessors have attempted to incorporate more of the data about the dose response curve by using benchmark dose (BMD) modeling. BMD modeling uses quantitative dose response models to estimate the dose that results in a specified change (such as 10\%) in the critical effect, or its precursor.

No human study involved exposures high enough to cause a decrease in $T_{4}$; therefore, all of the human studies can be said to have identified "freestanding NOAELs" for the critical effect. The highest NOAEL identified in the body of human studies is approximately $0.5 \mathrm{mg} / \mathrm{kg}$-day. This dose was achieved in workers exposed for an average of 8 years ${ }^{3,44}$ and in healthy adults exposed for 14 days in a clinical study. ${ }^{10}$ The lowest NOAEL observed in a human study ${ }^{45}$ is an estimated NOAEL of 0.006 $\mathrm{mg} / \mathrm{kg}$-day (actual exposure is an average of $0.112 \mathrm{mg} / \mathrm{L}$ ) measured in school-age children who had been exposed in utero and for their entire lifetime (about 7 years). Because, these children were exposed in utero and as neonates, the NOAEL from this study is a freestanding NOAEL in a sensitive population. Therefore, a NOAEL of $0.5 \mathrm{mg} / \mathrm{kg}$-day could be considered as a point-of-departure for the general human population, while $0.006 \mathrm{mg} / \mathrm{kg}$-day could be a point-of-departure for a sensitive human population.

However, use of a freestanding NOAEL does incorporate some uncertainty into the risk assessment because the true threshold for the critical effect has not been identified. In other words, the true threshold, or true NOAEL, is likely to be higher than the NOAEL used as the point-of-departure. 
Therefore, using this freestanding NOAEL would result in a more conservative risk value.

The Greer et $a l .{ }^{10}$ study adequately characterizes the dose-response curve for inhibition of iodine uptake in humans. This effect of perchlorate is a key event of the mode of action because it is the essential step in the cascade leading to adverse effects. Without inhibition of iodine uptake, there will be no alteration of $\mathrm{T}_{4}$ or TSH or subsequent adverse effects on neurological development and thyroid hyperplasia. Therefore, a point-of-departure based on inhibition of iodine uptake is a health-protective surrogate that can be used to replace a freestanding NOAEL for decreased $T_{4}$. The lowest dose evaluated by Greer et al., ${ }^{10} 0.007 \mathrm{mg} / \mathrm{kg}$-day, did not cause a statistically significant inhibition of iodine uptake. Based on a regression analysis taking into account the variability of the experimental population, the authors predicted that the dose that would result in $0 \%$ inhibition of iodine uptake is $0.0064 \mathrm{mg} / \mathrm{kg}$-day; the $95 \%$ upper confidence limit on iodine uptake inhibition at this dose is $8.3 \%$. Greer et al. ${ }^{10}$ concluded that an iodine uptake inhibition less than $10 \%$ would not be biologically significant. This threshold of $0.006 \mathrm{mg} / \mathrm{kg}$-day, as well as the lowest dose evaluated by Greer et al. ${ }^{10} 0.007 \mathrm{mg} / \mathrm{kg}$-day, are reasonable point-of-departures for estimating an $\operatorname{RfD}^{48,55}$

Currently, insufficient data exist to adequately define the level of iodine uptake inhibition in humans that can be tolerated for a lifetime without altering serum $\mathrm{T}_{4}$ and TSH levels. Greer et al. ${ }^{10}$ demonstrated that for 14-day exposure, inhibition of iodine uptake up to about $70 \%$, has no effect on serum $\mathrm{T}_{4}$ or TSH. Occupational studies ${ }^{3,44}$ demonstrated that workers exposed to perchlorate for several years demonstrated no altered $\mathrm{T}_{4}$ or TSH serum levels. When the serum hormone levels from these studies are plotted against serum perchlorate AUC predicted by the human PBPK model, it can be seen that chronic exposure in workers had no effect on serum $\mathrm{T}_{4}$ or TSH at serum AUC values that resulted in approximately 50\% I uptake inhibition. ${ }^{55}$ Thus, it might be reasonable to conclude that an appropriate benchmark response would be the perchlorate dose that resulted in a $50 \%$ inhibition of iodine uptake. Nonetheless, benchmark response levels of $10 \%$ inhibition of iodine uptake was modeled in order to be public health protective and take into account the uncertainties involved in extrapolating data from healthy adults to potential sensitive populations such as iodine deficient people, pregnant women, and neonates.

Based on BMD modeling results from Strawson et al. ${ }^{55}$ the perchlorate dose that caused a $10 \%$ inhibition of iodine uptake is $0.01 \mathrm{mg} / \mathrm{kg}$-day (BMD10); the its $95 \%$ lower limit (BMDL10) estimate ranges from $0.004 \mathrm{mg} / \mathrm{kg}$-day (Hill model) to $0.008 \mathrm{mg} / \mathrm{kg}$-day (Power model). These results are consistent 
with the conclusions of Greer et al., ${ }^{10}$ which indicated that the no effect level for iodine inhibition ranges from 0.006 (predicted) to 0.007 (measured) $\mathrm{mg} / \mathrm{kg}$-day.

Therefore, for the purpose of developing a perchlorate RfD, four different points-of-departure could have been used: a freestanding NOAEL of 0.5 $\mathrm{mg} / \mathrm{kg}$-day for the general, healthy population, a freestanding NOAEL of $0.006 \mathrm{mg} / \mathrm{kg}$-day for a sensitive subpopulation; the lowest dose evaluated by Greer et al., ${ }^{10} 0.007 \mathrm{mg} / \mathrm{kg}$-day; and a threshold for iodine uptake inhibition of $0.006 \mathrm{mg} / \mathrm{kg}$-day used as a health-protective surrogate for the freestanding NOAELs.

\section{Choice of uncertainty factors}

The judgment of the appropriate uncertainty factor is based on a review of the information supporting the choice of critical effect, and issues associated with extrapolation from experimental animals to humans and to sensitive humans. The noncancer risk assessment by U.S. EPA ${ }^{51}$ incorporates five different uncertainty factors to address issues of variability and uncertainty. Interspecies and intraspecies factors are used to address the uncertainty between experimental animals and humans, and the variability within different human populations. Three other factors (Subchronic, LOAEL, Database) are used to address lack of information. Typically, the maximum total uncertainty factor that U.S. EPA will apply is 3000 . If all five areas of uncertainty/variability are present warranting a total UF of 10,000 , then U.S. $\mathrm{EPA}^{51}$ generally concludes that the uncertainty is too great to develop an RfD.

\section{Interspecies Variability $\left(U F_{A}\right)$}

This factor accounts for the differences that occur between animals and humans and is also thought to be composed of subfactors for toxicokinetics (how the body distributes and metabolizes the chemical) and toxicodynamics (how the body responds to the chemical). If no information is available on the quantitative differences between animals and humans, then a default value of 10 is used. If information is available on one of the two subcomponents, then this information (chemical specific adjustment factor, CSAF) is used along with a default value of 3 for the remaining subfactor. ${ }^{58}$ If data are available to adequately describe variability in both subfactors, then actual data may be used to replace default values. If an RfD is based on human data, then a value of 1 is appropriate for this factor.

The body of data in experimental animals demonstrates that the rodent response to perchlorate is dramatically different than the human response. In 
rats, doses that cause only about $10 \%$ iodine uptake inhibition cause variable, but statistically significant changes in hormone levels. While in humans, doses that cause $70 \%$ iodine uptake inhibition have no effect on hormone levels. Basing the RfD on animal data will introduce greater uncertainty to the RfD than use of human data. Therefore, human data is the best basis for the RfD. If the proposed points-of-departure which are obtained from human studies are used, a factor of 1 is appropriate for this area of uncertainty. Otherwise, an uncertainty factor as high as 10 should be applied when animal data are used as the point-of-departure.

\section{Intraspecies Variability $\left(U F_{H}\right)$}

This factor accounts for the natural differences that occur between human subpopulations and for the fact that some individuals may be more sensitive than the average population. Similar to the interspecies uncertainty factor, this factor is also composed of two subfactors - one to account for toxicokinetic differences and one to account for toxicodynamic differences. If no information is available on human variability, then a default value of 10 is used. However, if adequate information is available on one or both of the two subcomponents, then this information is used along with a default value of 3 for the remaining subfactor. ${ }^{58}$ In addition, if an RfD is based on human data gathered in the known sensitive subpopulation, a value of less than 10 , perhaps even 1, may be chosen for this factor.

The judgment of appropriate intraspecies uncertainty factor depends in part on the choice of study as the basis of the RfD. For perchlorate exposure, the available data are insufficient to develop a CSAF for human variability at this time. When the RfD is based on the freestanding NOAEL of $0.5 \mathrm{mg} / \mathrm{kg}$ day identified in the healthy adult population, ${ }^{10}$ a full factor of 10 is appropriate to use because this NOAEL does not account for the fact that a NOAEL in sensitive subgroups (i.e., children or pregnant mothers with their fetuses) could be lower. In contrast, a lower factor is appropriate for the freestanding NOAEL of $0.006 \mathrm{mg} / \mathrm{kg}$-day identified in childre ${ }^{45}$ because this study covers at least one of the sensitive subpopulation, children. In the Crump et $a .^{45}$ study, the presence of perchlorate in the water has been a longterm problem. The mothers of the children evaluated were exposed before pregnancy, so that if perchlorate were affecting thyroid function in these women, they would already be hypothyroid at the start of pregnancy. The children themselves were exposed as fetuses in the uteri, as neonates, and throughout their lifetimes. Therefore several of the life stages that are considered sensitive have been studied in the Crump et al. ${ }^{10}$ study. Therefore, the observation of a freestanding NOAEL in this study gives greater confidence that fetuses, neonates, and children will be protected by an RfD based on this point-of-departure. However, an uncertainty factor of 3 , 
rather than 1 , is appropriate to use with this point-of-departure because there are no data to suggest how the other sensitive subpopulation, e.g., pregnant women, may respond.

When iodine uptake inhibition is used as a point-of-departure, the use of an uncertainty factor should be based on data addressing the variability of iodine uptake inhibition in human population. Mattie et $a l^{59}$ have used physiologically based pharmacokinetic models for both rats and humans to predict perchlorate doses that will result in a $5 \%$ iodine uptake inhibition in different life stages. In rats, the predicted doses that result in a $5 \%$ inhibition are $0.03 \mathrm{mg} / \mathrm{kg}$-day, $0.05 \mathrm{mg} / \mathrm{kg}$-day, and $0.13 \mathrm{mg} / \mathrm{kg}$-day for male rats, pregnant rats, and lactating rats, respectively. In humans, the predicted doses that result in a $5 \%$ inhibition are $0.01 \mathrm{mg} / \mathrm{kg}$-day, $0.025 \mathrm{mg} / \mathrm{kg}$-day, and $0.061 \mathrm{mg} / \mathrm{kg}$-day for healthy adult males and females, pregnant women, and lactating women, respectively. This analysis suggests that pregnant women are not more sensitive to iodine uptake inhibition than healthy adults. In addition, it confirms that the physiology of pregnancy serves to conserve iodine uptake, making pregnant women less sensitive to iodine uptake inhibition than non-pregnant adults. Thus, Strawson et al ${ }^{55}$ concluded that no extra uncertainty factor is necessary when iodine uptake inhibition data from the human study is used as a point-of-departure to derive an RfD. However, the $\mathrm{NRC}^{10}$ recommended use of a full factor of 10 to protect the most sensitive population, the fetuses of pregnant women who might have hypothyroidism or iodide deficiency. The NRC committee viewed its recommendation as conservative and health-protective because the point of departure is based on a non-adverse effect that precedes the adverse effect in the continuum of possible effects of perchlorate exposure (see Figure 1).

Therefore, the appropriate choice for intraspecies uncertainty factor is either 10 -fold with the use of the Greer et al. ${ }^{10}$ NOAEL for $\mathrm{T}_{4}$ decrease in adults, 3fold with the use of the Crump et al. ${ }^{45}$ NOAEL for $\mathrm{T}_{4}$ decrease in children, or 10 fold with the use of the Greer et al. ${ }^{10}$ threshold for iodine uptake inhibition.

\section{Subchronic to Chronic Extrapolation $\left(U F_{S}\right)$}

Because the RfD protects for a lifetime exposure, this factor is applied when the database lacks information on the health effects of the chemical following a chronic exposure. If the database contains no information on chronic exposure, a default value of 10 is often applied, unless other data suggest a lack of progression with exposure duration. If the database contains adequate chronic bioassays, then a value of 1 is generally appropriate. If there are data addressing only one of the two issues, then a default of 3 may be applied. Thus, the need for a duration UF for perchlorate depends on 
whether more sensitive effects are expected after increasing duration of exposure, or whether longer durations of exposure increase the severity or decrease the point of departure for perchlorate's critical effect.

While there are no studies that cover a full lifetime in either animals or humans for the thyroid effects of concern, there are studies that evaluate longer exposures in humans and studies that demonstrate no increase in the severity of effects with increasing duration in animals. In Gibbs et al. (1998) study, workers' tenure ranged from 1 to 27 years, with an average of 8 years. In Lamm et $a l^{44}$ study, $40 \%$ of the workers had a tenure greater than 5 years. In Crump et al. ${ }^{45}$ study, children age 6-8 years who had been exposed their entire lives were evaluated. In all three of these studies parameters investigated include general physical exam, tests of kidney and liver function, and blood counts, as well as tests of thyroid function. No effects on any of these parameters were observed in the exposed populations in these studies. When compared to the results of the 14-day clinical studies in humans, ${ }^{10,42,43}$ these longer-term studies show that increasing duration of exposure in humans does not increase the incidence or severity of thyroid effects, nor does it induce effects in other target organs that were not identified by the short-term studies. Although only preliminary data are available, a study in which volunteers were given 0.007 and $0.04 \mathrm{mg} / \mathrm{kg}$-day perchlorate for 6 months resulted in no effects on thyroid function and further supports these conclusions.

The available animal studies also support the conclusion that increasing exposure duration does not result in an increase in incidence or severity of thyroid effects nor does it reveal non-thyroid effects that are not detected by shorter-term studies. Several studies have evaluated perchlorate after either 14 days ${ }^{15,16,17,60}$ or 90 days. ${ }^{16,17,60}$ These studies have evaluated systemic and immunotoxic effects in addition to thyroid effects. None of these studies observed any non-thyroid effects after either 14 or 90 days of exposure, suggesting that increased exposure duration will not result in systemic effects that occur at lower doses than thyroid effects. Although the thyroid response is variable, particularly the hormone changes, these studies also show that animals exposed for 90 days do not show a clear pattern of more severe hormone changes nor an accelerated progression of thyroid pathology to hyperplasia compared with animals exposed for 14 days.

Strawson et al. ${ }^{55}$ also reviewed duration affect on inhibition of iodine uptake by perchlorate in animals and humans. Rats up-regulate iodine uptake very quickly during 14-day treatment and that inhibition actually decreases with time. ${ }^{9}$ In contrast, humans do not up-regulate iodine uptake within the same time period. ${ }^{10}$ However, these data do show that iodine uptake inhibition does not increase with increasing duration in either rats or humans. 
Animals or humans exposed to perchlorate for a prolong duration may develop other effects which didn't occur in short-tem studies, e.g., thyroid adenomas in F1 generation male rats of a two-generation study. ${ }^{27}$ However, it is known that thyroid tumors in rats are ultimately caused by constant stimulation of the thyroid by TSH. It is also known that perchlorate at 30 $\mathrm{mg} / \mathrm{kg}$-day caused dramatic increases in TSH in these animals. Thus, it is not necessarily surprising that tumors were evoked at such a high level of exposure. Nevertheless, the development of thyroid tumors in rats is not a duration effect per se, but rather a threshold phenomenon. If perchlorate doses stay below a level that induce increased TSH levels, then the production of thyroid tumors is not possible according to the proposed mode of action (Figure 2). ${ }^{21,47}$ Increased duration of perchlorate at doses that are below this threshold will not increase the risk of thyroid tumor formation. In addition, while the development of thyroid tumors in rats can be considered to be qualitatively relevant to humans, there are questions about whether humans do, in fact, develop thyroid tumors by the same mechanism.
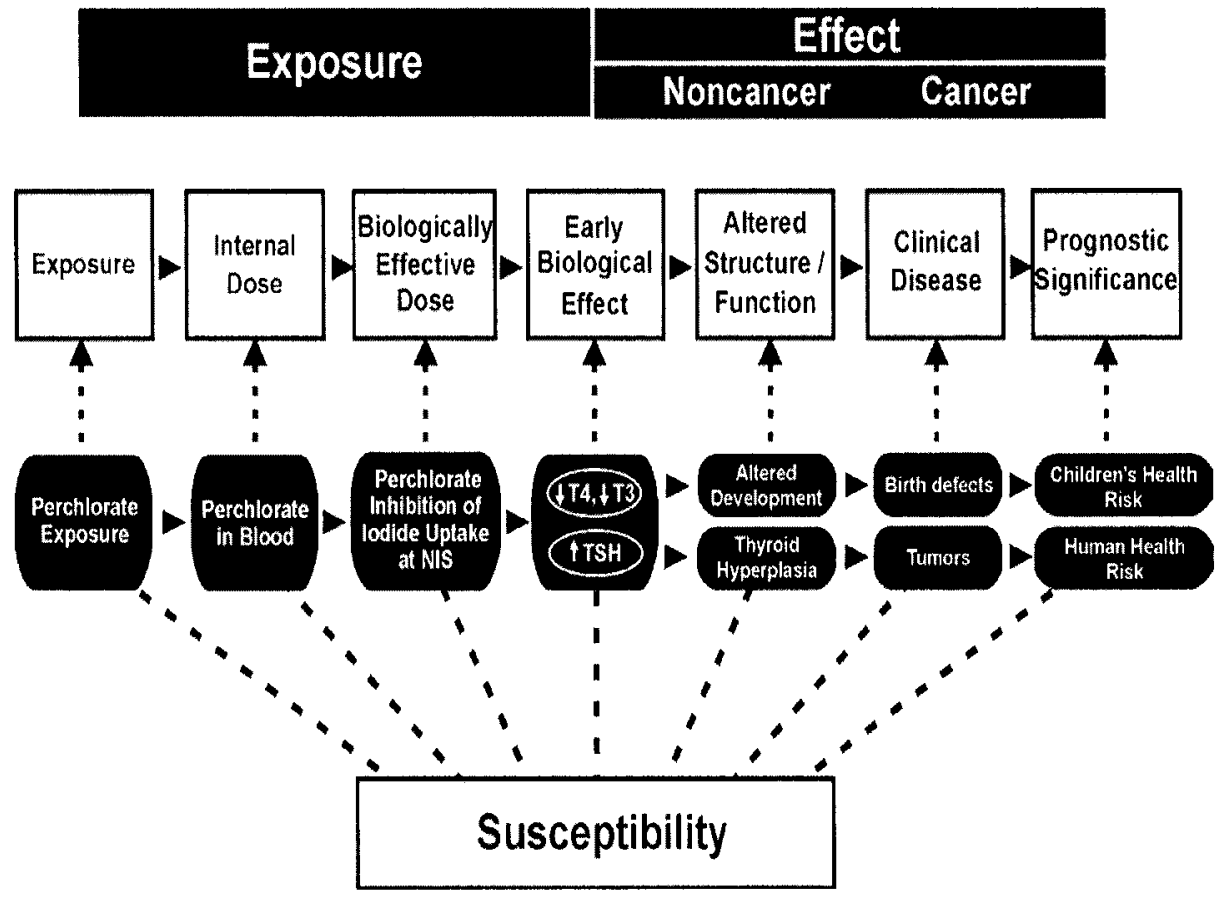

Figure 2. Mode of action model for perchlorate toxicity proposed by U.S. EPA (2003b). Perchlorate interferes with the sodium (NA+)-iodide (I-) symporter (NIS) present in various tissues, particularly thyroid. The model shows the exposure-dose response continuum considered in the context of biomarkers (classified as measures of exposure, effect, and susceptibility) and level of organization at which toxicity is observed (adapted directly from U.S. EPA. ${ }^{21}$ 
Based these considerations, a value of 1 appears to be appropriate to address this area of uncertainty. Both the human and animal studies demonstrate that increasing exposure duration does not result in the appearance of non-thyroid effects at doses lower than the thyroid hormone effects. Thyroid effects in humans and rodents do not increase in incidence or severity with increasing exposure duration. Inhibition of iodine uptake does not increase in humans or rats with increasing exposure duration. The $\mathrm{NRC}^{48}$ agreed that this uncertainty factor should be 1 .

\section{LOAEL to NOAEL Extrapolation (UF $)$}

Because the RfD is considered to be a subthreshold value that protects against any adverse health effects, this factor is applied when the database lacks information to identify a NOAEL. If the database does not identify a NOAEL, then a default of 10 is used for this factor. If a NOAEL is used, a value of 1 is appropriate. Often, if the database does not identify a NOAEL, but the adverse effects observed are of minimal severity, then a default of 3 will be considered appropriate for use of a "minimal LOAEL".

Both the Greer et al. ${ }^{10}$ and the Crump et al. ${ }^{45}$ studies identified freestanding NOAELs for the critical effect of decreased $T_{4}$. When either of these NOAELs is used as the point-of-departure for the development of an RfD, an uncertainty factor of 1 for this area would be appropriate. A point-ofdeparture at the threshold for iodine uptake inhibition (the lowest dose in Greer et al. $)^{10}$ is, likewise, not considered to be a LOAEL, because inhibition of iodine uptake is a key event in the mode of action rather than an adverse effect. $^{48}$ In addition, this point of departure represents a dose at which no inhibition of iodine uptake occurs, so that adverse effects cannot occur following exposure to this dose. This conclusion is confirmed by the body of human data, which demonstrate that no effect on serum hormone levels has been observed at doses equal to or higher than this point of departure. Therefore, this point-of-departure should be considered as a NOAEL surrogate, rather than a LOAEL surrogate, and the appropriate value for this factor is 1 . The $\mathrm{NRC}^{10}$ agreed that this uncertainty factor should be 1 .

\section{Database $\left(U F_{D}\right)$}

Based on US EPA's risk assessment methodology, the database for deriving a high confidence RfD includes at a minimum two chronic bioassays by the appropriate route of exposure in different species, one two-generation reproductive toxicity study, and two developmental toxicity studies in different species. The minimal database required for deriving an RfD is a single subchronic bioassay, that includes a full histopathology examination. The database factor is used to account for the fact that a potential health effect may not be identified if the database is missing a particular type of 
study. This factor may also be used if the existing data indicate the potential for a heath effect that is not fully characterized by the standard bioassays, for example neurotoxicity or immunotoxicity. If the database is complete, a value of 1 is appropriate. If only the minimal database is available, then a default of 10 is used. A value of 3 may be used if the database is missing one or two key studies.

The database for perchlorate includes an large number of experimental animal studies, including chronic (but older) studies that show tumors at high doses, ${ }^{61}$ numerous shorter-term bioassays that unequivocally demonstrate that thyroid disturbance occurs at lower doses than other systemic, immunotoxic, genotoxic, or other effects, developmental toxicity studies in two species, a 2 generation reproduction study that also monitored systemic effects in young rats, a developmental neurotoxicity study, a specialized developmental toxicity study to monitor hormone changes in early life and during late pregnancy and lactation, and a specialized neurobehavioral study to confirm earlier findings. The database also includes human clinical, experimental, epidemiology, and occupational studies.

All of this information demonstrates that the thyroid is the most sensitive organ system. In humans, the threshold for iodine uptake inhibition is well characterized and additional studies are not likely to provide different information that would change the risk assessment. In humans, the perchlorate dose that causes a decrease in $T_{4}$, the critical effect, is not well characterized since no human population has been exposed to a dose high enough to alter hormone levels. However, if these studies could be performed, their effect would likely be to raise the NOAEL. The mode of action analysis suggests a potential for adverse effects as a result of serum $T_{4}$ levels that are consistently depressed by at least $60 \%$. The doses that cause this degree of $\mathrm{T}_{4}$ decrease are not well characterized in either humans or animals. However, by selecting a point-of-departure that is below the threshold for any $T_{4}$ change, subsequent effects will not develop. Therefore, the overall perchlorate database is complete, and any new studies that are conducted to fine tune our knowledge of the perchlorate mode of action will not identify lower points-of-departure than can be estimated from the existing database. Based on these considerations, the appropriate value for this factor is 1 .

In summary, when human data are used as the point-of-departure, the only area of uncertainty for a perchlorate RfD that needs to be addressed by the use of uncertainty factors is human variability and the difference in response between pregnant women and the groups for which data are available. A factor of 1 is appropriate to address all other areas of uncertainty. The NRC committee ${ }^{48}$ recommended the use of a composite uncertainty factor of 10 to 
protect the fetuses of pregnant women who might have hypothyroidism or iodide deficiency.

\section{Developing the RfD}

As shown by extensive animal studies, the critical effect of perchlorate is $T_{4}$ serum decrease. Pregnant rats are demonstrated to be the most sensitive subgroup, likely followed by the young rat. Several human studies exist that monitored for this critical effect. These human studies do not include pregnant women, but they do include children. In addition, comparative data between the experimental animal and human indicate that humans are not more sensitive than the experimental animal species tested to $T_{4}$ serum decrease by perchlorate; in fact based on toxicodynamics parameters they are much less sensitive. ${ }^{56}$ The most relevant data for developing the RfD for perchlorate exposures comes from human epidemiology and clinical studies, supplemented with available and extensive information on experimental animals. All these data support the use of the human data for development of an $\mathrm{RfD}^{48}$

The NRC Committee report, ${ }^{48}$ "Health Implications of Perchlorate Ingestion," recommended using the data from the Greer study for deriving a reference dose (RfD) for perchlorate. Animal and clinical studies conducted since 1997 were all designed based on the mechanism of action for perchlorate, inhibition of iodide uptake. This mechanism of action has been a point of agreement between toxicologists and risk assessors throughout the process to develop the perchlorate risk assessment. There remains disagreement on what is the first adverse or critical effect after perchlorate exposure. The NRC committee avoided all disagreements by using the NOEL for inhibition of iodide uptake from the Greer study as the point of departure when they recommended an RfD for perchlorate. According to the NRC report, ${ }^{48}$ there is an added level of conservatism or protection, since inhibition of iodide uptake is the first step in a multi-step process prior to causing an adverse effect. The NRC committee ${ }^{48}$ recommended use of a composite uncertainty factor of 10 to protect the fetuses of pregnant women who might have hypothyroidism or iodide deficiency. The US Environmental Protection Agency (USEPA) agreed and developed their RfD based on the NRC recommendation $(0.007 \mathrm{mg} / \mathrm{kg}$-day point-of-departure $\mathrm{x}$ UF of 10$)$. The USEPA listed the oral reference dose (RfD) for perchlorate as 0.0007 $\mathrm{mg} / \mathrm{kg}$-day (24.5 ppb drinking water equivalent) in their Integrated Risk Information System (IRIS). 


\section{SUMMARY AND IMPLICATIONS}

There are currently many unanswered questions surrounding the prevalence of perchlorate in the environment, the possible exposures and effects, and the risk management strategies available to manage perchlorate contamination. Further information must be obtained in each of these areas in order to develop successful and appropriate strategies for handing perchlorate contamination now and in the future. Because of the uncertainty involved in evaluating toxicological data, the states and federal agencies using this information may use and interpret it somewhat differently to promulgate different standards and advisory levels. It is important to note that the status of the knowledge of perchlorate exposure and risks continues to evolve and it will be essential to periodically re-evaluate what is known about this chemical. However, the reference dose recommended by National Research Council and adopted by the U.S. Environmental Protection Agency will protect the health of even the most sensitive groups of people over a lifetime of exposure.

\section{ACKNOWLEDGMENTS}

The author wishes to acknowledge all of the researchers who contributed to the perchlorate database and the Department of Defense, Perchlorate Study Group and NASA for funding perchlorate research. Special thanks go to Teresa R. Sterner for assistance with the preparation of this chapter.

\section{REFERENCES}

1. Motzer, W.E. Perchiorate: Problems, detection, and solutions. Environ. Forensics. 2001; 2:301-311.

2. Urbansky, E.T. Perchlorate chemistry: implications for analysis and remediation. Bioremed. J. 1998; 2:81-95.

3. Gibbs, J.P., Ahmad, R., Crump, K.S., Houck, D.P., Leveille, T.S., Findley, J.E., Francis, M. Evaluation of a population with occupational exposure to airborne ammonium perchlorate for possible acute or chronic effects on thyroid function. J Occup. Environ. Med. 1998; 40:1072-1082.

4. Braverman, L.E., He, X., Pino, S., Cross, M., Magnani, B., Lamm, S.H., Kruse, M.B., Engel, A., Crump, K.S., and Gibbs, J.P. The effect of perchlorate, thiocyanate, and nitrate on thyroid function in workers exposed to perchlorate long-term. The Journal of Clinical Endocrinology \& Metabolism. 2005; 90(2):700-706.

5. Greenspan, F.S. The role of fine-needle aspiration biopsy in the management of palpable thyroid nodules. Am. J. Clin. Pathol. 1997; 108:S26-30. 
6. Scanlon, M.F, Issa, B.G., Dieguez, C. Regulation of growth hormone secretion. Horm. Res. 1996; 46(4-5): 149-54.

7. Capen, C.C. Mechanistic data and risk assessment of selected toxic end points of the thyroid gland. Toxicol. Pathol. 1997; 25:39-48.

8. Wolff, J. Perchlorate and the thyroid gland. Pharmacolog. Rev. 1998; 50:89-105.

9. Yu, K.O., Narayanan, L., Mattie, D.R., Godfrey, R.J., Todd, P.N., Sterner, T.R., Mahle, D.A., Lumpkin, M.H., Fisher, J.W. 2002. The pharmacokinetics of perchlorate and its effect on the hypothalamus- pituitary-thyroid axis in the male rat. Toxicol. Appl. Pharmacol. 2002; 182:148-159.

10. Greer, M.A., Goodman, G., Pleus, R.C., Greer, S.E. Health effects assessment for environmental perchlorate contamination: The dose response for inhibition of thyroidal radioiodine uptake in humans. Environ. Health Perspect. 2002; 110:927-937.

11. Merrill, E.A., Clewell, R.A., Gearhart, J.M., Robinson, P.J., Sterner, T.R., Yu, K.O., Mattie, D.R., Fisher, J.W. PBPK predictions of perchlorate distribution and its effect on thyroid uptake of radioiodide in the male rat. Toxicol. Sci. 2003; 73:256-269.

12. Merrill, E.A., Clewell, R.A., Robinson, P.J., Jarabek, A.M., Gearhart, J.M., Sterner, T.R., Fisher, J.W. PBPK model for radioactive iodide and perchlorate kinetics and perchlorate-induced inhibition of iodide uptake in humans. Toxicol. Sci. 2005; 83:25-43.

13. Clewell, R.A., Merrill, E.A., Yu, K.O., Mahle, D.A., Sterner, T.R., Mattie, D.R., Robinson, P.J., Fisher, J.W., Gearhart, J.M. Predicting fetal perchlorate dose and inhibition of iodide kinetics during gestation: A physiologically-based pharmacokinetic analysis of perchlorate and iodide kinetics in the rat. Toxicol. Sci. 2003a; 73:235-255.

14. Clewell, R.A., Merrill, E.A., Yu, K.O., Mahle, D.A., Sterner, T.R., Fisher, J.W., Gearhart, J.M. Predicting neonatal perchlorate dose and inhibition of iodide uptake in the rat during lactation using physiologically-based pharmacokinetic modeling. Toxicol. Sci. $2003 b ; 74: 416-436$.

15. Caldwell, D.J., King, J.H., Kinkead, E.R., Wolfe, R.E., Narayanan, L., Mattie, D.R. Results of a fourteen day oral-dosing toxicity study of ammonium perchlorate. In: 1995 JANNAF Safety and Environmental Protection Subcommittee Meeting: Volume 1, Tampa, FL. Joint Army, Navy, NASA, Air Force (JANNAF) Interagency Propulsion Committee Publication 634. Columbia, MD: Chemical Propulsion Information Agency. 1995.

16. Siglin, J.C., Mattie, D.R., Dodd, D.E., Hildebrandt, P.K., Baker, W.H. A 90-Day drinking water toxicity study in rats of the environmental contaminant ammonium perchlorate. Toxicol. Sci. 2000; 57:61-74.

17. Keil, D., Warren, D.A., Jenny, M., EuDaly, J., Dillard, R. Effects of ammonium perchlorate on immunotoxicological, hematological, and thyroid parameters in $\mathrm{B} 6 \mathrm{C} 3 \mathrm{~F} 1$ female mice. Medical University of South Carolina, Charleston, SC. Final report of contract DSWA01-97-0008. 1999 
18. Narayanan, L. Consultative letter, AFRL-HE-WP-CL-2000-0034. Thyroid hormone and TSH co-laboratory study report [memorandum with attachments to Annie Jarabek]. Wright-Patterson Air Force Base, Dayton, OH: Air Force Research Laboratory. June 15, 2000 .

19. York, R.G., Lewis, E., Brown, W.R., Girard, M.F., Mattie, D.R., Funk, K.A. and Strawson, J.S. 2005a. Refining the Effects Observed in a Developmental Neurobehavioral Study of Ammonium Perchlorate Administered Orally in Drinking Water to Rats. I - Thyroid and Reproductive Effects (In Press).

20. York, R.G., Barnett, Jr, J., Girard, M.F., Mattie, D.R., Bekkedal, M., Garman, R.H. and Strawson, J.S. 2005b. Refining the Effects Observed in a Developmental Neurobehavioral Study of Ammonium Perchlorate Administered Orally in Drinking Water to Rats. II - Behavioral and Neurodevelopment Effects (In Press).

21. U.S. Environmental Protection Agency. Disposition of Comments and Recommendations for Revisions to "Perchlorate Environmental Contamination: Toxicological Review and Risk Characterization External Review Draft (January 16, 2002)." National Center for Environmental Assessment, Washington, DC. October 27, 2003a.

22. York, R.G., Brown, W.R., Girard, M.F., Dollarhide, J.S. Oral (drinking water) developmental toxicity study of ammonium perchlorate in New Zealand white rabbits. Int. J. Toxicol. 2001a; 20:199-205.

23. York, R.G., Funk, K.A., Girard, M.F., Mattie, D., Strawson, J.E. Oral (drinking water) developmental toxicity study of ammonium perchlorate in Sprague-Dawley rats. Int. J. Toxicol. 2003; 22:453-464.

24. York, R.G., Barnett, J., Brown, W.R., Garman, R.H., Mattie, D.R., Dodd, D. A rat neurodevelopmental evaluation of offspring, including evaluation of adult and neonatal thyroid, from mothers treated with ammonium perchlorate in drinking water. Int. J. Toxicol. 2004; 23:191-214.

25. Bekkedal, M.Y.V., Carpenter, T., Smith, J., Ademujohn, C., Maken, D., Mattie, D.R. A neurodevelopmental study of the effects of oral ammonium perchlorate exposure on the motor activity of pre-weanling rat pups. Naval Health Research Center Detachment (Toxicology), Wright-Patterson AFB, OH. TOXDET-00-03, 2000.

26. Bekkedal, M.Y.V., Arffsten, D., Mattie, D. An evaluation of neurobehavioral tests used to assess the neurodevelopmental effects of early ammonium perchlorate exposure. J. Toxicol. Environ. Health A. 2004; 67:835-844.

27. York, R.G., Brown, W.R., Girard, M.F., Dollarhide, J.S. Two-generation reproduction study of ammonium perchlorate in drinking water in rats evaluates thyroid toxicity. Int. J. Toxicol. 2001b; 20:183-197.

28. Soldin, O.P., Braverman, L.E., Lamm, S.H. Perchlorate clinical pharmacology and human health: a review. Ther. Drug Monit. 2001; 23:316-331.

29. Lamm, S.H., and M. Doemland. Has perchlorate in drinking water increased the rate of congenital hypothyroidism? J. Occup. Environ. Med. 1999; 41(5):409-411. 
30. Li, Z., F.X. Li, D. Byrd, G.M. Deyhle, D.E. Sesser, M.R. Skeels, and S.H. Lamm. Neonatal

thyroxine level and perchlorate in drinking water. J. Occup. Environ. Med. 2000; 42(2):200-205.

31. Li, F.X., D.M. Byrd, G.M. Deyhle, D.E. Sesser, M.R.Skeels, S.R.Katkowsky, and S.H. Lamm. Neonatal Thyroid-Stimulating Hormone Level and Perchlorate in Drinking Water. Teratology. 2000; 62(6):429-431.

32. Li, F.X., L. Squartsoff, and S.H. Lamm. Prevalence of thyroid diseases in Nevada counties with respect to perchlorate in drinking water. J. Occup. Environ. Med. 2001; 43(7):630634.

33. Chang, S., C. Crothers, S. Lai, and S. Lamm. Pediatric neurobehavioral diseases in Nevada counties with respect to perchlorate in drinking water: An ecological inquiry. Birth Defects Res. Part A Clin. Mol. Teratol. 2003; 67(10):886-892.

34. Brechner, R.J., Parkhurst, G.D., Humble, W.O., Brown, M.B., Herman, W.H. Ammonium perchlorate contamination of Colorado River drinking water is associated with abnormal thyroid function in newborns in Arizona. J. Occup. Environ. Med. 2000; 42:777-782.

35. Schwartz, J. Gestational Exposure to Perchlorate is Associated with Measures of Decreased Thyroid Function in a Population of California Neonates. M.S. Thesis, University of California, Berkeley. 2001.

36. Kelsh, M.A., P.A. Buffler, J.J. Daaboul, G.W. Rutherford, E.C. Lau, J.C. Cahill, A.K. Exuzides, A.K. Madl, L.G. Palmer, and F.W. Lorey. Primary congenital hypothyroidism, newborn thyroid function, and environmental perchlorate exposure among residents of a southern California community. J. Occup. Environ. Med. 2003; 45(10):1116-1127.

37. Lamm, S. H. Perchlorate exposure does not explain differences in neonatal thyroid function

between Yuma and Flagstaff. [Letter]. J. Occup. Environ. Med. 2003; 45(11):1131-1132.

38. Buffler, P.A., M.A. Kelsh, E.C. Lau, C.H. Edinboro, and J.C. Barnard. 2004. Epidemiologic Studies of Primary Congenital Hypothyroidism and Newborn Thyroid Function Among California Residents, Final Report. April 2004, Berkeley, CA.

39. Morgan, J.W., and R.E. Cassady. 2002. Community cancer assessment in response to long-time exposure to perchlorate and trichloroethylene in drinking water. J. Occup. Environ. Med. 44(7):616-621.

40. Brabant, G., Bergmann, P., Kirsch, C.M., Kohrle, J., Hesch, R.D., von zur Muhlen, A. Early adaptation of thyrotropin and thyroglobulin secretion to experimentally decreased iodine supply in man. Metabolism. 1992; 41:1093-1096.

41. Braverman, L.E., X. He, S. Pino, B. Magnani, and A. Firek. The effect of low dose perchlorate on thyroid function in normal volunteers [abstract]. Thyroid. 2004; 14(9):691.

42. Lawrence, J.E., Lamm, S.H., Pino, S., Richman, K., Braverman, L.E. The effect of shortterm low-dose perchlorate on various aspects of thyroid function. Thyroid. 2000;10:659663. 
43. Lawrence, J., Lamm, S., Braverman, L.E. Low dose perchlorate (3 mg daily) and thyroid function. Thyroid 2001; 11:295.

44. Lamm, S.H., Braverman, L.E., Li, F.X., Richman, K., Pino, S., Howearth, G. Thyroid health status of ammonium perchlorate workers: A cross- sectional occupational health study. J. Occup. Environ. Med. 1999; 41:248-260.

45. Crump, C., Michaud, P., Tellez, R., Reyes, C., Gonzalez, G., Montgomery, E.L., Crump, K.S., Lobo, G., Becerra, C., Gibbs, J.P. Does perchlorate in drinking water affect thyroid function in newborns or school-age children? J. Occup. Environ. Med. 2000; 42:603612.

46. Tellez, R.T., Chacon, P.M., Abarca, C.R., Blount, B.C., Van Landingham, C.B., Crump, K.S., and Gibbs, J.P. Chronic Environmental Exposure to Perchlorate Through Drinking Water and Thyroid Function During Pregnancy and the Neonatal Period. Thyroid. 2005; (In Press).

47. Hill, R.N., Erdreich, L.S., Paynter, O.E., Roberts, P.A., Rosenthal, S.L., Wilkinson, C.F. Thyroid follicular cell carcinogenesis. Fundam. Appl. Toxicol. 1989; 12:629-697.

48. National Research Council. 2005a. Health Implications of Perchlorate Ingestion. Washington, D.C.: National Academies Press.

49. Barnes, D.G., Dourson, M.L. Reference dose (RfD): Description and use in health risk assessments. Reg. Toxicol. Pharmacol. 1988; 8:471-486.

50. Dourson, M.L. Methods for establishing oral reference doses. In: Risk Assessment of Essential Elements. W. Mertz, C. O. Abernathy, and S. S. Olin, eds. Washington, D.C.: ILSI Press. Ch. 51-61. 1994.

51. U.S. Environmental Protection Agency. A Review of the Reference Dose and Reference Concentration Processes. Risk Assessment Forum. Washington, DC. May 2002. EPA/630/P-02/002A.

52. U.S. Environmental Protection Agency. Integrated Risk Information System (IRIS). Glossary of Terms. Office of Research and Development. Washington, DC. 2003b. Available online: http://www.US EPA.gov/iris.

53. Haber, L.T., Dollarhide, J.S., Maier, A., Dourson, M.L. Noncancer Risk Assessment: Principles and Practice in Environmental and Occupational Settings. In Patty's Toxicology. E. Bingham, B. Cohrssen and C.H. Powell Ed. 5th ed, Wiley and Sons, Inc. New York, NY. p. 169-232. 2001.

54. University of Nebraska. Perchlorate State of the Science Symposium. University of Nebraska Medical Center, Omaha, Nebraska. September 29th to October 1, 2003. Available online: http://www.unmc.edu/coned.

55. Strawson, J., Zhao,Q., Dourson, M. Reference dose for perchlorate based on thyroid hormone change in pregnant women as the critical effect. Regul. Toxicol. Pharmacol. 2004; 39(1):44-65. 
56. Capen, C.C. Toxic responses of the endocrine system. In: Casarett and Doull's Toxicology The Basic Science of Poisons. $6^{\text {th }}$ edition. C. D. Klaassen, ed. New York: McGraw-Hill. Ch. 21. pp. 724. 2001.

57. Toxicology Excellence for Risk Assessment (TERA). Use of human data in risk assessment. Comments submitted to U.S. EPA. February 19, 2002.

58. Meek, M., Renwick, A., Ohanian, E., Dourson, M., Lake, B., Naumann, B., Vu, V. Guidelines for application of compound specific adjustment factors (CSAF) in dose/concentration response assessment. Comm. Toxicol. 2001; 7:575-590.

59. Mattie, D.R., Sterner, T.R., Merrill, E.A., Clewell, R.A., Zhao, Q., Strawson, J.E., Dourson, M.L. Use of Human and Animal PBPK Models in Risk Assessment for Perchlorate. The Toxicologist. 2004; 78(S-1): 361.

60. BRT. Ammonium perchlorate: Effect on immune function. Quality assurance audit: study no. BRT 19990524 - plaque forming cell (PFC) assay; study no. BRT 19990525 - local lymph node assay (LLNA) in mice. Burleson Research Technologies, Inc., Raleigh, NC. Study \# BRT 19990524 \& 19990525. 2000.

61. Kessler, F.J., Krüskemper, H.J. (1966). Experimentelle Schilddrüsentumoren durch mehrjährige Zufuhr von Kaliumperchlorat [Experimental thyroid tumors caused by longterm potassium perchlorate administration]. Klin. Wochenschr. 1966; 44:1154-1156. 